\title{
Design of an inexpensive integrating sphere laboratory setup for the optical characterization of a light source
}

Frédéric Leloup, Sven Leyre, Toon Van den Abeele, Peter Hanselaer

Frédéric B. Leloup, Sven Leyre, Toon Van den Abeele, Peter Hanselaer, "Design of an inexpensive integrating sphere laboratory setup for the optical characterization of a light source," Proc. SPIE 9793, Education and Training in Optics and Photonics: ETOP 2015, 979313 (8 October 2015); doi: $10.1117 / 12.2223098$

Event: Education and Training in Optics and Photonics: ETOP 2015, 2015, Bordeaux, France 


\title{
Design of an inexpensive integrating sphere laboratory setup for the optical characterization of a light source
}

\author{
Frédéric B. Leloup*, Sven Leyre, Toon Van den Abeele, Peter Hanselaer \\ Light \& Lighting Laboratory, Dept. of Electrical Engineering (ESAT), KU Leuven, Gebroeders De \\ Smetstraat 1, B-9000 Gent, BELGIUM
}

\begin{abstract}
Since about five years, Lighting has become a partly required and partly elective course within the Energy program of the Master of Engineering Technology at KU Leuven. While the theoretical part of the course is lectured to the entire audience, an increased emphasis has been placed on an individual evaluation of the students for the laboratory module. In order to admit several students simultaneously to the laboratory, multiple constructions of the same laboratory setup are requested. Therefore, cheap alternatives to the scientific metrology instrumentation, which still guarantee that the students get acquainted with optical metrology techniques and general radiometric and photometric quantities, are needed. In this paper, the design of an inexpensive integrating sphere setup is presented, enabling the optical characterization of light sources. Instead of using an expensive sphere with magnesium oxide or barium sulfate coating, a cheap polystyrene sphere is employed. In combination with a low-cost USB spectroradiometer, the system enables the direct measurement of the spectral radiant power of a light source. In addition, the luminous flux, luminous efficacy, colour coordinates, colour temperature, and colour rendering index can be determined. The equipment used, the experimental procedure, as well as some typical measurement results are presented.
\end{abstract}

Keywords: optical metrology, optics education, integrating sphere, radiometry, photometry, colorimetry

\section{INTRODUCTION}

KU Leuven is Belgium's largest university. Founded in 1425, KU Leuven has been a centre of learning for nearly six centuries, being one of the oldest universities in Europe. As a leading European research university and co-founder of the League of European Research Universities, KU Leuven offers a wide variety of international master programmes, all supported by innovative, interdisciplinary research. Besides the faculties of Science, Engineering Science, Bioengineering Science, and Architecture, the Faculty of Engineering Technology is one of the five faculties within the Science, Engineering \& Technology Group. It gathers all Industrial Sciences programmes at seven locations throughout Flanders.

Within the Energy programme of the Master of Engineering Technology, courses on Optics and Lighting are partly required and partly elective. While the theoretical part of these courses is lectured to the entire audience, an increased emphasis has been placed on an individual supervision and evaluation of the students for the laboratory module. This way, students are able to gain a deeper understanding of specific topic areas of radiometry, photometry, and colorimetry. In order to admit several students simultaneously to the laboratory, multiple constructions of the same laboratory setup are requested. Therefore, cheap alternatives to the scientific metrology instrumentation, which still guarantee that the students get acquainted with optical metrology techniques and general radiometric and photometric quantities, are needed. This paper reports on the design of an inexpensive integrating sphere setup, enabling the optical characterization of light sources. Besides the equipment used, the experimental procedure as well as some typical measurement results are described.

\section{THEORETICAL BACKGROUND}

The integrating sphere is the most common and widely used device for measuring optical radiation. ${ }^{1}$ It is designed to achieve a homogeneous distribution of the optical radiation by means of multiple Lambertian reflections at the sphere's inner surface. When used for measuring the radiant power and total luminous flux of a light source, the integrating

*frederic.leloup@kuleuven.be; phone +32 9 265.87.13; fax +32 9 225.62.69; lichttechnologie.be

Education and Training in Optics and Photonics: ETOP 2015, edited by Eric Cormier, Laurent Sarger Proc. of SPIE Vol. 9793, 979313 · @ 2015 SPIE, IEEE, OSA, ICO · doi: 10.1117/12.2223098 
sphere behaves as the input optical element for a detector of radiant power, placed at the exit port of the sphere. Depending on the type of emission of the light source ( $2 \pi$ vs. $4 \pi$ steradian), the source can either be positioned in front of the sphere's entrance port or inside the sphere, respectively.

Under the ideal circumstances of a spherical geometry with infinitesimally small entrance and exit ports, and a sphere coating which behaves as a perfectly homogeneous Lambertian reflector with a total reflectance $\rho$ being wavelength independent, the relation between the light flux of the source $\phi_{\text {source }}$ and the receiver illuminance $E_{\text {receiver }}$ is defined as

$$
E_{\text {receiver }}=\frac{1}{4 \pi R^{2}} \cdot \frac{\rho}{1-\rho} \cdot \phi_{\text {source }}=f . \phi_{\text {source }},
$$

with $R$ the sphere radius, $f$ being defined as the sphere factor. ${ }^{2}$

Traditionally, specific coating materials such as barium sulfate $\left(\mathrm{BaSO}_{4}\right)$ or magnesium oxide $(\mathrm{MgO})$ are utilized for the sphere coating due to their high diffuse reflectance in the visible and near-infrared range of the spectrum (up to $99 \%$ ), and due to their thermal stability until elevated temperatures of up to $350{ }^{\circ} \mathrm{C}$, depending on the type of coating. ${ }^{3}$

Even by using this type of materials, in practice the ideal conditions cannot be met. For example, the reflectance value of the bottom part of the sphere will usually be lower due to contamination of the sphere coating with dust. Moreover, the surface of the detector itself can be considered as a deviation of the uniform diffuse coating requirement. Without any adaptations to the sphere geometry, the receiver also detects a part of the light emitted by the source directly. This contribution is not taken into account in the calculation of Eq. 1, and it is dependent on the luminance of the light source in the direction of the detector. As such, it will vary with the orientation of the source in the sphere (except for real isotropic sources). Therefore, the detector must be baffled against direct irradiation by the light source. Finally, also the light source under test and its accessories cause a source of measurement error.

It must therefore be concluded that accurate absolute radiant power and total luminous flux measurements by use of an integrating sphere are not evident, since Eq. 1 is not valid in practice, and corrections are required. Yet, if the intensity distribution of two light sources is more or less identical, one can expect that the proportionality between the measured

illuminance $E_{\text {receiver }}$ and the light flux $\phi_{\text {source }}$ still holds. As such, by application of the so-called 'substitution method', in which first a reference source with known light flux $\phi_{\text {ref }}$ is measured, followed by the light source under test, the flux of the test source $\phi_{\text {test }}$ can be derived according to

$$
\phi_{\text {test }}=\phi_{\text {ref }} \cdot \frac{E_{\text {test }}}{E_{\text {ref }}},
$$

with $E_{r e f}$ and $E_{\text {test }}$ representing the measured illuminance of the reference source and test source, respectively.

In practice correction methods are introduced, such as the use of an auxiliary lamp or more elaborated methods as proposed by Ohno and Zong. ${ }^{4}$ A detailed description of these correction methods falls however out of the scope of this paper.

\section{EXPERIMENTAL SETUP}

The goal of the integrating sphere laboratory assignment is for the students to characterize the optical and electrical performance of a set of light sources. In order to understand the measurement procedure, the theoretical background as briefly discussed in section 2 is instructed in the theoretical course. Furthermore, students have to hand in a written assignment, in which they are asked to calculate and to compare the luminous efficacy of two different light sources, before and as a preparation for the laboratory.

Instead of using an expensive research laboratory-based integrating sphere setup, five measurement setups were constructed by use of cheap polystyrene spheres of diameter $50 \mathrm{~cm}$. As an example, a picture of one of the setups is provided in Fig. 1, while a schematic overview of the entire measurement system is presented in Fig. 2.

The lower part of the sphere is fixed to a table holder, while the upper part of the sphere can be retrieved in order to insert the light source. A hole is drilled into the top of the upper half of the sphere, through which the wiring is allowed 


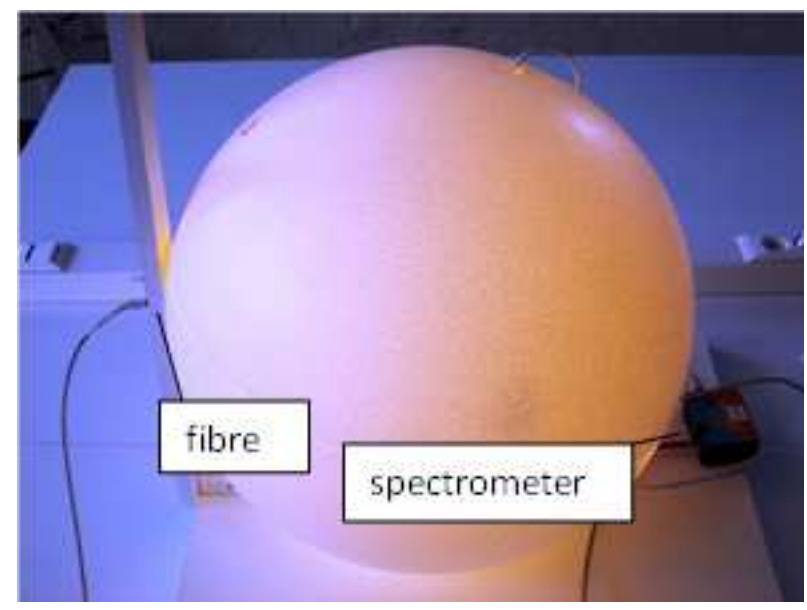

Figure 1. Picture of the polystyrene integrating sphere setup, including the optical fibre (Ocean Optics type QP200-2-VISBX) coupled to the USB-650 Red Tide spectrometer.

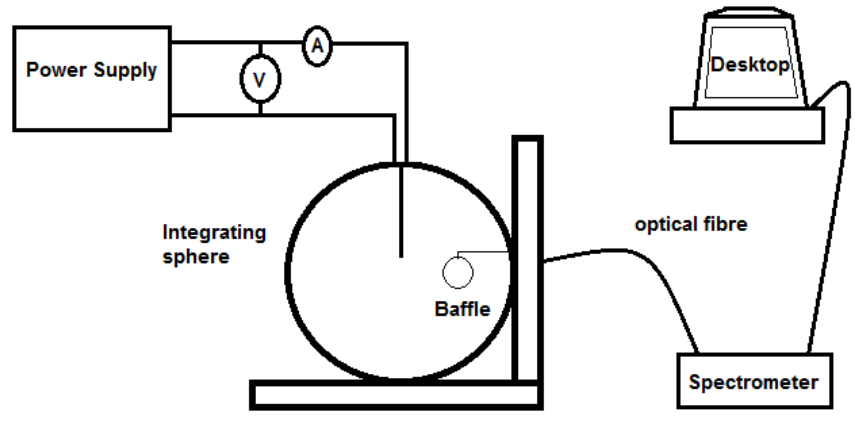

Figure 2. Schematic overview of the experimental integrating sphere setup.

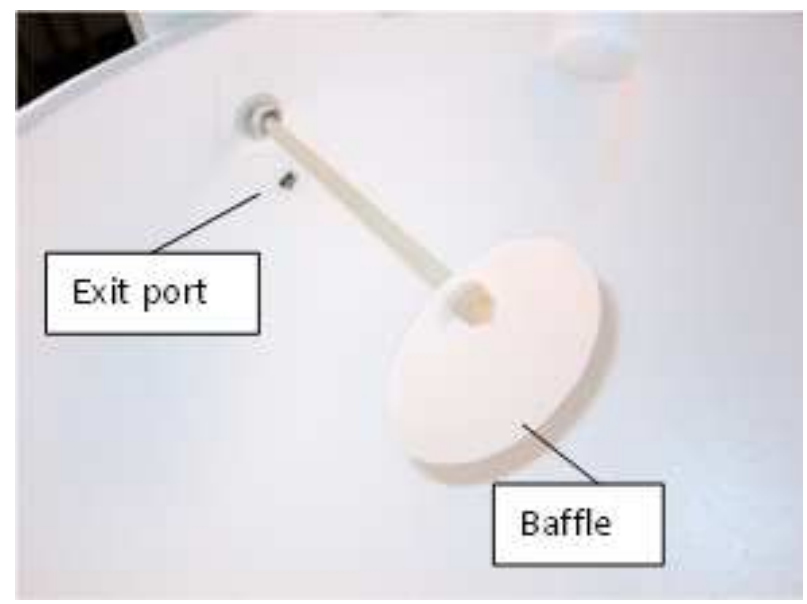

Figure 3. Inside the integrating sphere a baffle is mounted to prevent direct incident light to enter the optical fibre detector.

Proc. of SPIE Vol. 9793 979313-3 
for driving the light source electrically by use of a power supply. A voltmeter and ammeter resp. record the voltage across and the current through the source under test.

A second whole is drilled into the side of the sphere in order to act as an exit port. A $2 \mathrm{~m}$ optical fibre with fibre core size of $200 \mu \mathrm{m}$ (Ocean Optics type QP200-2-VIS-BX) is coupled to this port via the table holder, through an SMA 905 connector. A second SMA 905 connector connects the other end of the fibre to a spectrometer (Ocean Optics USB-650 Red Tide spectrometer, wavelength range $350-1000 \mathrm{~nm}$ ). This preconfigured spectrometer is coupled to a desktop computer through a USB cable. Inside the sphere, a baffle (diameter $8 \mathrm{~cm}$ ) is mounted in front of the sphere, preventing direct incident light to enter the detector (see Fig. 3).

Eventually, a fibre splitter can be used to lower the cost of the setup. A fibre splitter is comprised of three fibres; two fibre legs on one side which overlap in a junction with a third fibre at the common end. This way, the signal acquisition of two sources, measured with two different integrating spheres, can be performed with just one spectrometer.

For the processing of the measurement data Logger Pro data-collection and analysis software is used. Alternatively, specific software tools for spectroscopy applications provided by the spectrometer manufacturer may be used (e.g. OceanView by Ocean Optics).

\section{EXAMPLE MEASUREMENT RESULTS}

As an example, the determination of the start-up time and the total luminous flux of a low-energy compact fluorescent lamp (CFL) denoted as 'Carrefour 11W' will further be discussed. To determine the total luminous flux of this test source, the substitution method as described in section 2 is put forward. As such, a reference light source has to be provided. In this case, an incandescent lamp (Sylvania GLS 60W 240 E27 Clear) with a theoretic total luminous flux of $700 \mathrm{~lm}$ was chosen for the purpose. The effective total luminous flux of the lamp was measured in-house by use of a near-field goniophotometer. ${ }^{5}$ Yet, if there is no possibility to measure the luminous flux of the reference lamp, the product details as provided by the lamp manufacturer could still be used.

Since the intensity distribution of the reference light source and the test bulb is more or less identical, one can expect that the proportionality between the measured illuminance and light flux holds. At first, the power $P_{\text {ref }}$ and the illuminance $E_{r e f}$ of the reference source with known total luminous flux $\phi_{r e f}$ are measured. Recorded values are presented in Table 1. From these values, the luminous efficacy $\eta_{r e f}(\mathrm{~lm} / \mathrm{W})$ of the reference source can be calculated according to Eq. 3:

$$
\eta_{\text {ref }}=\frac{\phi_{\text {ref }}}{P_{\text {ref }}}
$$

This value numbers $11.6 \mathrm{~lm} / \mathrm{W}$.

Table 1. Determination of the power $P_{r e f}(\mathrm{~W})$ and illuminance $E_{r e f}(\mathrm{lux})$ of the reference lamp with known luminous flux $\phi_{r e f}$ $(\operatorname{lm})$.

\begin{tabular}{|l|l|l|}
\hline$\phi_{\text {ref }}(\mathbf{l m})$ & $\boldsymbol{P}_{\text {ref }}(\mathbf{W})$ & $\boldsymbol{E}_{\text {ref }}(\mathbf{l u x})$ \\
\hline 693.4 & 59.6 & 360 \\
\hline
\end{tabular}

Afterwards, the test source is installed. The power $P_{\text {test }}$ and illuminance $E_{\text {test }}$ of the test source are now recorded each $15 \mathrm{~s}$ for a total period of 6 minutes. The measurement results are provided in Table 2. From these results, it can be concluded that the illuminance increases for about $4 \mathrm{~min}$, after which it stabilizes. The final illuminance after 6 min numbers 336 lux. According to Eq. 2 and Eq. 3, this results in a total luminous flux and a luminous efficacy of the test lamp of $647 \mathrm{~lm}$ and $57 \mathrm{~lm} / \mathrm{W}$, respectively. 
Table 2. Determination of the power $P_{\text {test }}(\mathrm{W})$ and illuminance $E_{\text {test }}(\mathrm{lux})$ of the 'Carrefour $11 \mathrm{~W}$ ' test source as a function of time (s).

\begin{tabular}{|c|c|c|}
\hline Time (s) & $\boldsymbol{P}_{\text {test }}(\mathbf{W})$ & $E_{\text {test }}(\mathbf{l u x})$ \\
\hline 15 & 10.4 & 70 \\
\hline 30 & 11.0 & 142 \\
\hline 45 & 11.2 & 207 \\
\hline 60 & 11.5 & 242 \\
\hline 75 & 11.5 & 255 \\
\hline 90 & 11.5 & 262 \\
\hline 105 & 11.5 & 270 \\
\hline 120 & 11.6 & 280 \\
\hline 135 & 11.7 & 293 \\
\hline 150 & 11.7 & 304 \\
\hline 165 & 11.7 & 314 \\
\hline 180 & 11.7 & 322 \\
\hline 195 & 11.8 & 330 \\
\hline 210 & 11.8 & 335 \\
\hline 225 & 11.8 & 339 \\
\hline 240 & 11.7 & 342 \\
\hline 255 & 11.7 & 343 \\
\hline 270 & 11.6 & 343 \\
\hline 285 & 11.6 & 342 \\
\hline 300 & 11.5 & 343 \\
\hline 315 & 11.5 & 343 \\
\hline 330 & 11.5 & 342 \\
\hline 345 & 11.4 & 338 \\
\hline 360 & 11.3 & 336 \\
\hline
\end{tabular}

\section{CONCLUSIONS}

In this paper, the design of an inexpensive laboratory setup for the measurement of the total luminous flux of light sources has been presented. Instead of using an expensive laboratory-based sphere, a polystyrene sphere was used in combination with a low-cost USB spectroradiometer. After a brief consideration of integrating sphere theory, the experimental design of the alternative setup was discussed. Finally, the measurement procedure was explained by use of some real measurement data.

While the uncertainty on the reported measurement results might be large, the goal of the described experiments is for the students to get acquainted with applications of radiometry and photometry, data collection, and data analysis, as in a professional context. A significant emphasis is therefore also placed on the correct scientific representation of the results 
in a laboratory report, to be handed in after having performed the experiments. With the described test setup, these targets are fulfilled. Furthermore, in this paper the reported optical properties of the light source were restricted to the start-up time, the total luminous flux and the luminous efficacy. Yet, it is clear that also radiometric properties, such as the radiant power, the colour temperature, and the colour rendering index, can be investigated with the same equipment. Depending on the type of students (bachelor or master degree), the assignment can perfectly be made modular.

\section{REFERENCES}

[1] Technical guide: Integrating Sphere Theory and Applications , Labsphere, http://www.labsphere.com/uploads/technical-guides/a-guide-to-integrating-sphere-theory-and-applications.pdf.

[2] Tutorial on light measurement, Gigahertz-Optik, http://www.light-measurement.com/tutorials-on-lightmeasurement/tutorials.pdf.

[3] Technical guide: Reflectance Materials and Coatings, Labsphere, http://www.labsphere.com/uploads/technicalguides/a-guide-to-reflectance-materials-and-coatings.pdf.

[4] Ohno, Y. and Zong, Y., "Detector-based integrating sphere photometry," Proc. CIE 133, 155-160 (1999).

[5] Audenaert, J., Acuna R., P. C., Hanselaer, P., and Leloup, F. B., "Practical limitations of near-field goniophotometer measurements imposed by a dynamic range mismatch," Opt. Express 23(3), 2240-2251 (2015). 